\title{
Asymptotics of near-cloaking
}

\author{
J.R. OCKENDON ${ }^{1}$, H OCKENDON ${ }^{1}$, B.D. SLEEMAN ${ }^{2,3}$ and R H TEW ${ }^{4}$ \\ ${ }^{1}$ Mathematical Institute, University of Oxford, \\ Oxford, OX2 6GG, UK \\ ${ }^{2}$ School of Mathematics, University of Leeds, \\ Leeds, LS2 9JT, UK \\ ${ }^{3}$ Division of Mathematics, University of Dundee, \\ Dundee, DD1 $4 H N$, UK \\ ${ }^{4}$ School of Mathematical Sciences, University of Nottingham, \\ University Park, Nottingham, NG7 2RD, UK
}

June 5, 2020

\begin{abstract}
This paper describes how asymptotic analysis can be used to gain new insights into the theory of cloaking of spherical and cylindrical targets within the context of acoustic waves in a class of linear elastic materials. In certain cases these configurations allow solutions to be written down in terms of eigenfunction expansions from which high-frequency asymptotics can be extracted systematically. These asymptotics are compared with the predictions of ray theory and are used to describe the scattering that occurs when perfect cloaking models are regularised.
\end{abstract}

\section{Introduction}

A widely-studied (Cummer et al [1], Greenleaf et al [2]) non-dimensional model for cloaking a sphere of radius $a$ insonified by plane acoustic waves is

$$
(r-a)^{2} \frac{\partial^{2} p}{\partial r^{2}}+2(r-a) \frac{\partial p}{\partial r}+\frac{1}{\sin \theta} \frac{\partial}{\partial \theta}\left(\sin \theta \frac{\partial p}{\partial \theta}\right)+k_{s h}^{2}(r-a)^{2} p=0,
$$

where $p$ is a complex function whose real part is the pressure, $(r, \theta)^{1}$ are spherical polar coordinates and the cloak lies in $a<r<b$; the cloak, or sheath, is characterised by the parameter $k_{s h}$. The region $r>b$ is a homogeneous medium in which wave propagation is described by the Helmholtz equation

$$
\nabla^{2} p+k_{0}^{2} p=0,
$$

\footnotetext{
${ }^{1}$ In view of the symmetry of the problem we assume that $p$ is independent of the azimuthal angle $\phi$.
} 
where $k_{0}=k_{s h}\left(\frac{b-a}{b}\right)$.

The cloak and its surroundings are coupled by the conditions

$$
[p]_{b^{+}}^{b^{-}}=0, \quad\left[\frac{1}{\rho} \frac{\partial p}{\partial r}\right]_{b^{+}}^{b^{-}}=0,
$$

where the density $\rho(r)$ is given by

$$
\rho= \begin{cases}1 & r>b \\ \left(\frac{b-a}{b}\right)\left(\frac{r}{r-a}\right)^{2} & b>r>a,\end{cases}
$$

and all variables are dimensionless.

The insonification is described by $e^{i k_{0} r \cos \theta}$ so that the total axisymmetric wavefield in $r>b$ is

$$
p=e^{i k_{0} r \cos \theta}+p_{s c},
$$

where $p_{s c}$ is the outgoing scattered field. Boundary conditions at $r=a$ may not need precise definition in view of the form of (1), but, if necessary, they can be homogeneous Dirichlet or Neumann conditions.

The above model has been constructed in such a way that the scattered field is zero and

$$
p= \begin{cases}e^{i k_{0} r \cos \theta} & r>b \\ e^{i k_{s h}(r-a) \cos \theta} & b>r>a,\end{cases}
$$

so that the target $r=a$ is perfectly cloaked for any value of $k_{0}$ and, by symmetry, for all angles of the incoming radiation. We will say more about its derivation shortly but we note at once that the density profile (4) is physically unrealistic at $r=a$ and one of our principal aims is to perturb some of the variables to avoid this shortcoming. We also note that (1) is a traditional Helmholtz equation after a 'transformation optics' shift in the radial space coordinate and we will also find that (6) still holds for cylindrical geometries. Indeed as indicated in references [1]- [6], transformation optics can be applied to many kinds of wave propagation and many different insonifications.

A physical derivation for the model has been described in detail in [1] (and references cited therein), whose notation we have adopted. The simplest physical realisation is that of elastic waves propagating in an inhomogeneous cloak which has the property of having negligible shear modulus ${ }^{2}$ compared to its isotropic bulk modulus which is highly inhomogeneous and takes the dimensionless form

$$
\lambda=\frac{(b-a)^{3} r^{2}}{(r-a)^{2} b^{3}}
$$

\footnotetext{
${ }^{2}$ This assumption is not unreasonable for certain rubber-like materials.
} 
as the shear modulus tends to zero. Moreover, it is crucial that the cloak density tensor $\underline{\underline{\rho}}$ is anisotropic, taking the constant value $\frac{b-a}{b}$ in the circumferential and azimuthal directions as distinct from (4) in the radial direction. As explained in Norris [4] and associated references, such a surprising property may be realised in certain metamaterials.

A detailed account of cloaking of elastic waves is given in [3] and [6] and a simple derivation of (1) is as follows.

Under the above assumptions, classical linear elastic wave propagation is modelled by a displacement $\boldsymbol{U}$ satisfying

$$
\underline{\underline{\rho}} \frac{\partial^{2} \boldsymbol{U}}{\partial t^{2}}=\nabla(\lambda \nabla \cdot \boldsymbol{U})+\mu\left(\nabla(\nabla \cdot \boldsymbol{U})+\nabla^{2} \boldsymbol{U}\right),
$$

where $\nabla^{2}=\nabla(\nabla \cdot)-\nabla \times(\nabla \times)$ and $\mu$ is the soon-to-be-neglected constant dimensionless shear modulus. Hence, in the frequency domain we write $\boldsymbol{U}=\operatorname{Re}\left(e^{-i \omega t} \boldsymbol{u}\right)$, and we now non-dimensionalise time in order to make $\omega=k_{0}$, so that

$$
\begin{gathered}
-\lambda \nabla \cdot \boldsymbol{u}=p, \\
-k_{0}^{2} \underline{\underline{\boldsymbol{\rho}}} \boldsymbol{u}+\nabla p+\mu\left(\nabla\left(\frac{p}{\lambda}\right)-\nabla^{2} \boldsymbol{u}\right)=\mathbf{0} .
\end{gathered}
$$

Multiplying by the inverse $\underline{\underline{\rho}}^{-1}$, taking the divergence and taking the singular limit as $\mu \rightarrow 0$, we obtain

$$
\frac{k_{0}^{2} p}{\lambda}+\nabla \cdot\left(\underline{\underline{\rho}}^{-1} \nabla p\right)=0
$$

Since $k_{s h}^{2}=\frac{k_{0}^{2} b^{2}}{(b-a)^{2}}$ and using (7), this model reduces to (1) when

$$
\underline{\underline{\rho}}=\left(\begin{array}{ccc}
\frac{(b-a)}{b} \frac{r^{2}}{(r-a)^{2}} & 0 & 0 \\
0 & \frac{(b-a)}{b} & 0 \\
0 & 0 & \frac{(b-a)}{b}
\end{array}\right)
$$

in spherical polar coordinates, assuming no azimuthal variation.

Typical boundary conditions for (11) are that the complex displacement $\boldsymbol{u}$ or the tractions $\underline{\underline{\boldsymbol{\tau}}} \cdot \boldsymbol{n}$ are prescribed at a boundary with normal $\boldsymbol{n}$, where $\underline{\underline{\boldsymbol{\tau}}}$ is the stress tensor

$$
\underline{\underline{\boldsymbol{\tau}}}=\lambda(\nabla \cdot \boldsymbol{u}) \underline{\underline{\boldsymbol{I}}}+2 \mu \underline{\underline{\boldsymbol{e}}},
$$

where $\underline{\underline{\boldsymbol{I}}}$ is the identity matrix and $\underline{\underline{\boldsymbol{e}}}$ is the strain tensor $e_{i j}=\frac{1}{2}\left(\frac{\partial u_{i}}{\partial x_{j}}+\frac{\partial u_{j}}{\partial x_{i}}\right)$ in Cartesian coordinates. Hence, when $\mu=0$, prescribing $\underline{\underline{\boldsymbol{\tau}}} \cdot \boldsymbol{n}$ is equivalent to prescribing $\lambda \nabla \cdot \boldsymbol{u}$ and zero traction corresponds to homogeneous Dirichlet data for $\nabla \cdot \boldsymbol{u}$ and hence $p$. Equally, using (10), zero displacement (i.e. a rigid target) corresponds to $\frac{\partial p}{\partial n}=0$. 
As explained in [4], the unrealistic values of $\underline{\underline{\boldsymbol{\rho}}}$ and $\lambda$ in (12), (7) as $r \downarrow a$ need not arise for cloaks such as 'pentamaterials' that have more general anisotropy. In this paper, we will avoid infinite parameter values by regularising the model so that the target is expanded from $r=a$ to $r=a(1+\epsilon)$ where $0<\epsilon \ll 1$. This is an example of what is called near-cloaking, see for example [7], [8], [9]. One of the aims of our paper is to complement this literature by deriving some specific asymptotic expansions of the solution in the physical plane.

We note that, without expanding the target, it is shown in $[4,5]$ that a radial scaling can reduce the problem to that of a classical Helmholtz equation in which $\underline{\underline{\rho}}$ and $\lambda$ are determined by the scaling; when the scaling is linear in $r,(1)$ is retrieved.

We make the important observation that the solution to the problem for the near-cloak can be obtained by writing down the classical solution, as described in [15] or [10], for a target of radius $a b \epsilon /(b-a)$, using transformation optics (TO) to define the variable

$$
r_{r o}=\frac{b(r-a)}{(b-a)}
$$

and solving the Helmholtz equation

$$
\nabla_{\text {ro }}^{2} p+k_{0}^{2} p=0 .
$$

Although this transformation holds for any values of the parameters $k_{0}$ and $\epsilon$, carrying out the asymptotic analysis in terms of these parameters in the TO plane is no easier than carrying out a direct asymptotic expansion on the model (1). Moreover, the physical interpretation of, for example, the shadow boundary is clearer when we work in physical space.

In Section 2 we will do this both for spherically symmetric configurations described by (1) and also for cylindrically symmetric targets. Then, in Section 3, we will apply ray theory to derive the solutions to all these problems directly when $k_{s h}$ is large. Several generalisations will be considered by the same methods in Section 4 .

We conclude this section by briefly recapitulating the classical separation of variables technique in terms of spherical Hankel functions $h_{n}^{(1)}$ and Legendre polynomials $P_{n}$, which results in a series expansion for solutions of (2) in the general form

$$
p(r, \theta)=\sum_{n=0}^{\infty} \alpha_{n} h_{n}^{(1)}\left(k_{0} r\right) P_{n}(\cos \theta),
$$

in which the $\alpha_{n}$ 's are constants. This expansion is useful for relatively small values of $k_{0}$ but for large $k_{0}$ it converges extremely slowly. Indeed, if $a$ is a typical scatterer dimension then, for a relative error of 1 per cent, $O\left(k_{0} a\right)$ terms of the series need to be computed. This difficulty is overcome by appealing to the 'Watson' transformation, which relies upon the identification of the sum as the residue evaluation of a strategically-chosen integral taken over a specified contour $C$. 
In general, the important identity is

$$
\int_{C} \frac{f(\nu)}{\sin (\pi \nu)} d \nu \sim 2 i \sum_{n=-N}^{N}(-1)^{n} f(n) \quad \text { as } \quad N \rightarrow \infty,
$$

where, for example, $C$ comprises two infinitely long parallel, straight lines - one lying infinitesimally below and parallel to the real $\nu$-axis and the other infinitesimally above it - and connected by infinitesimally short, straight lines parallel to the imaginary $\nu$-axis 'at infinity' (along which both contributions to the integral vanish in the limit), with $C$ being traversed in a counter-clockwise sense. The basic idea is that within this infinitesimally thin contour, the only poles of the integrand on the left hand side of (15) are the integer zeroes of $\sin (\pi \nu)$. When the contour is deformed to encompass the complex poles of $f(\nu)$, then, when we use the methods of stationary phase and steepest descents to evaluate the integral asymptotically, the resulting residue series is found to be rapidly convergent and only the first term of the residue series is needed to secure sufficient accuracy. For further details, see Jones [10] or Spence [11].

\section{Exact solutions for spherical and cylindrical cloaks}

\subsection{Spherical cloaks}

We begin by considering the way in which the solution for a spherical target $r=a$ can be found by separating the variables.

\subsubsection{Target $r=a$}

We assume that in the spherical polar region $r>b$ a plane wave

$$
p(r, \theta)=p_{\text {inc }}(r, \theta)=e^{i k_{0} r \cos \theta}
$$

insonifies the cloak externally, giving rise to a scattered field $p_{s c}(r, \theta)$ within the same region and a corresponding pressure field $p(r, \theta)$ inside the cloak $(a \leq r \leq b)$. Our procedure will be closely related to that used for uncloaked spheres (see [15] or Ch 8 in [10]) for which (15) is a key ingredient.

Noting the Jacobi-Anger expansion (Colton and Kress [14])

$$
e^{i k_{0} r \cos \theta}=\sum_{n=0}^{\infty} i^{n}(2 n+1) j_{n}\left(k_{0} r\right) P_{n}(\cos \theta),
$$

where $j_{n}$ is a spherical Bessel function, we are motivated to write down expressions for the outgoing scattered field $p_{s c}$ and the field $p$ within the cloak in the forms

$$
p_{s c}(r, \theta)=\sum_{n=0}^{\infty} \alpha_{n} i^{n}(2 n+1) h_{n}^{(1)}\left(k_{0} r\right) P_{n}(\cos \theta), \quad r>b
$$


and

$$
p(r, \theta)=\sum_{n=0}^{\infty} i^{n}(2 n+1)\left[\beta_{n} j_{n}\left(k_{s h}(r-a)\right)+\gamma_{n} h_{n}^{(1)}\left(k_{s h}(r-a)\right)\right] P_{n}(\cos \theta), \quad a<r<b,
$$

where $h_{n}^{(1)}$ is the spherical Hankel function of the first kind. Thus, the total field in the region $r \geq b$ exterior to the cloak is

$$
\begin{aligned}
p_{t}(r, \theta) & =p_{\text {inc }}(r, \theta)+p_{s c}(r, \theta) \\
& =\sum_{n=0}^{\infty} i^{n}(2 n+1)\left[j_{n}\left(k_{0} r\right)+\alpha_{n} h_{n}^{(1)}\left(k_{0} r\right)\right] P_{n}(\cos \theta),
\end{aligned}
$$

and we note that this expression can also be used when satisfying more general boundary conditions. Notice that the 'scattered' component $p_{s c}$ depends only upon the specific spherical Hankel function of the first kind $h_{n}^{(1)}\left(k_{0} r\right)$; this choice guarantees that the scattered field $p_{s c}$ radiates away from the boundary $r=b$ as $r \rightarrow \infty$.

We note that applying the boundary conditions (3) on $r=b$ yields the algebraic equations

$$
\begin{aligned}
\beta_{n} j_{n}\left(k_{0} b\right)+\gamma_{n} h_{n}^{(1)}\left(k_{0} b\right) & =j_{n}\left(k_{0} b\right)+\alpha_{n} h_{n}^{(1)}\left(k_{0} b\right) \\
\beta_{n} j_{n}^{\prime}\left(k_{0} b\right)+\gamma_{n} h_{n}^{(1)^{\prime}}\left(k_{0} b\right) & =j_{n}^{\prime}\left(k_{0} b\right)+\alpha_{n} h_{n}^{(1)^{\prime}}\left(k_{0} b\right)
\end{aligned}
$$

where we recall that $k_{0}=k_{s h}(b-a) / b$, and these must both be satisfied regardless of any conditions imposed upon $p$ either within the cloak $a<r<b$ or on its inner boundary $r=a$. Since the Wronskian

$$
W\left(k_{0}, b\right)=j_{n}\left(k_{0} b\right) h_{n}^{(1)^{\prime}}\left(k_{0} b\right)-j_{n}^{\prime}\left(k_{0} b\right) h_{n}^{(1)}\left(k_{0} b\right)=\frac{i}{k_{0}^{2} b^{2}},
$$

we see that $\alpha_{n} \equiv \gamma_{n}$ and $\beta_{n} \equiv 1$ for each non-negative integer $n$.

We now have a choice as to how to proceed. One option is to adopt the approach taken in [1], which is to insist upon the field being everywhere finite within the cloak, including at the inner boundary. This choice necessitates $\gamma_{n} \equiv 0$, from which $\alpha_{n} \equiv 0$ then follows whilst $\beta_{n} \equiv 1$ remains true from the previous analysis. The solution in the cloak can then be summed explicitly using (16) to give the exact solution (6). The price that is paid for perfect cloaking is the presence of unphysical singularities in (7) and (12), together with the inability to impose specific and realistic boundary condition on the target $r=a$. Nonetheless, it is interesting that the assumption that the field is finite at $r=a$ generates a physically acceptable reflected field within the cloak.

In the light of these comments our strategy here, is to maintain every detail of the cloaking material, including its mathematical description, but to relocate the target at a slightly 
displaced position specified by $r=a(1+\epsilon),(0<\epsilon \ll 1)$ within the cloak. Thus, regardless of which of the spherical Bessel functions arise in the solutions that follow, all are now guaranteed to remain finite within and on any boundary to the solution domain. Unfortunately, this approach will never allow the perfect cloaking achieved previously. Even so, it is interesting to calculate the radiation back into the exterior region $r \geq b$ and estimating this loss is one of our objectives.

We remark that this 'near-cloaking' configuration for spheres and cylinders has been addressed using transformation optics in [4] and [5] where numerical results are derived for $K=\mathcal{O}(1)$ and asymptotic results for $K \rightarrow 0$.

\subsubsection{Target $r=a(1+\epsilon)$}

The boundary conditions at $r=b$ still hold, as do the general solutions (18) and (19), and so the previous conclusions that $\alpha_{n}=\gamma_{n}, \beta_{n} \equiv 1$ remain valid. However, rather than

insisting upon finite fields in $a \leq r \leq b$, we now impose either a Neumann $\left(\frac{\partial p}{\partial r}=0\right)$ or Dirichlet $(p=0)$ boundary condition at $r=a(1+\epsilon)$. Setting $K=k_{s h} a \epsilon$, these then lead to the additional constraints

$$
\begin{array}{ll}
\text { Neumann: } & j_{n}^{\prime}(K)+\gamma_{n} h_{n}^{(1)^{\prime}}(K)=0 \\
\text { Dirichlet: } & j_{n}(K)+\gamma_{n} h_{n}^{(1)}(K)=0,
\end{array}
$$

and so the solutions are completely fixed with

$$
\alpha_{n}=\gamma_{n}=-j_{n}^{\prime}(K) / h_{n}^{(1)^{\prime}}(K)
$$

for Neumann data or

$$
\alpha_{n}=\gamma_{n}=-j_{n}(K) / h_{n}^{(1)}(K)
$$

for Dirichlet data.

Our next step is to analyse the corresponding infinite series solution asymptotically by an application of a Watson transformation of the type referred to in the Introduction, and the calculation proceeds as follows.

First, we note that the series solutions for the spherical geometry under review involve eigenfunction expansions summed over non-negative integer orders. In this case, the integration path $C$ referred to in (15) is replaced by a 'keyhole' contour $\Gamma$ running from infinity towards zero immediately below the real $-\nu$ axis and then returning to infinity along another straight path, this time running immediately above the real- $\nu$ axis. The path encircles the origin in such a way that the 'loop' connecting the upper and lower branches of $\Gamma$ crosses the real axis at $\nu=\nu_{0}$ lying somewhere between -1 and 0 (in order to avoid capturing a contribution from $n=-1$ or other negative values of $n$ further along the negative real axis). Notice that the sense of integration along this path, which is chosen so that we ultimately 
end up with integrals along the real- $\nu$ axis (or, technically, just above it) which run from 'minus' to 'plus' infinity, is now no longer traversed in a counter-clockwise sense necessitating a minus sign on the right-hand side of (15).

This integration path is not convenient for subsequent asymptotic analysis, for which we need to replace the lower branch of $\Gamma$ by another which runs just above the negative real axis and which connects continuously with the original upper branch of $\Gamma$. Referring to the eigenfunction expansions once more and for simplicity considering Neumann data prescribed on the target at $r=a(1+\epsilon)$, we see that appropriate choices for the function $f(\nu)$ relevant to the total field evaluated (a) on the target boundary itself and (b) within the observation region $r \geq b$ are:

$$
\begin{aligned}
f(\nu) & =-\frac{1}{2 K^{2}} \frac{e^{-i \nu \pi / 2}(2 \nu+1)}{h_{\nu}^{(1)^{\prime}}(K)} P_{\nu}(\cos \theta), \\
f(\nu) & =\frac{i}{2} e^{-i \nu \pi / 2}(2 \nu+1) \frac{\left[j_{\nu}\left(k_{0} r\right) h_{\nu}^{(1)^{\prime}}(K)-j_{\nu}^{\prime}(K) h_{\nu}^{(1)}\left(k_{0} r\right)\right]}{h_{\nu}^{(1)^{\prime}}(K)} P_{\nu}(\cos \theta),
\end{aligned}
$$

and we note that $h_{\nu}^{(1)^{\prime}}(K)$ has no real zeros. Connection formulae in $\nu$ can then be used in both cases (26) and (27) to establish the invariance of $f(\nu)$ under the transformation $\nu \rightarrow-(\nu+1)$, with continuity of the integration path described above being guaranteed by the universal choice $\nu_{0}=-\frac{1}{2}$ (so that $\nu_{0}=-\left(\nu_{0}+1\right)$ ). This now leads to an integration path $D$ as shown in Figure 1 .

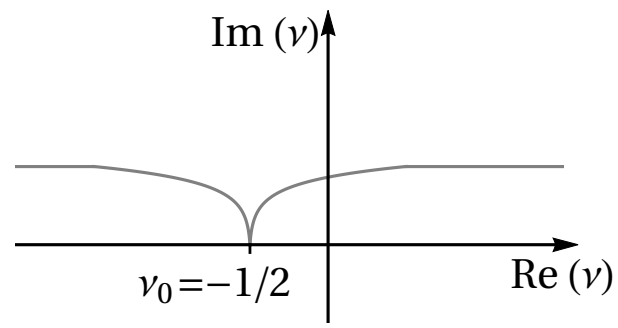

Figure 1: The integration contour $D$

The analysis of the integral representation of the solution resulting from the use of the functions (26) or (27) is dependent on whether we consider it in the geometrical shadow (where the incoming field is blocked by the impeding spherical target and cloak) or in the illuminated region accessible to the incident irradiation. In both spatial regions, we can think in terms of decomposing the total field into the following constituents: (i) the incident field, (ii) the "geometrical optics" field and (iii) the diffracted field. In the shadow, the geometrical optics field exactly cancels the incident field leaving just the diffracted field as the leading-order contribution, whereas, in the illuminated region, the geometrical optics field supplies the specularly reflected wave, and the diffracted wave co-exists with both this 
and the incident field but, as we shall see, at an exponentially lower order.

Concentrating on the reflected field in the illuminated region, our first step is to write the Legendre function $P_{\nu}(\cos \theta)$ in $(27)$ in the form

$$
P_{\nu}(\cos \theta)=e^{i \nu \pi} P_{\nu}(-\cos \theta)-i e^{i \nu \pi} R_{\nu}^{0}(\cos \theta) \sin (\nu \pi)
$$

([13], Chapter 10) where, in terms of standard associated Legendre functions,

$$
R_{\nu}^{0}(\cos \theta)=P_{\nu}(\cos \theta)+\frac{2 i}{\pi} Q_{\nu}(\cos \theta)
$$

and from which, as long as $\theta$ is not close to 0 or $\pi$,

$$
R_{\nu}^{0}(\cos \theta) \sim\left(\frac{2}{\pi \nu \sin \theta}\right)^{\frac{1}{2}} e^{-i\left[\left(\nu+\frac{1}{2}\right) \theta-\frac{\pi}{4}\right]} \quad \text { as } \quad \nu \rightarrow \infty
$$

follows. The denominator $\sin (\nu \pi)$ in (15) is maintained with regard to the first term on the right-hand side of (28), and the corresponding integral can be evaluated by deforming the contour $\Gamma$ to encompass the poles arising from the zeroes of $h_{\nu}^{(1)^{\prime}}(K)$, which can be expressed in terms of an Airy function. When the resulting integral is evaluated as a residue series using the method of stationary phase, we find that the field in the shadow is exponentially small as $K \rightarrow \infty$; we return to this in Section 3.1.2. The $\sin (\nu \pi)$ term in the denominator disappears altogether when considering the contribution from the second term in (28) and the asymptotic representation of this part of the solution contains the geometrical optics field we are interested in calculating. In fact, after some labour this entire contribution (which we now label $p_{g o}$ ) to the (so far exact) integral representation of the total field in $r \geq b$ can be expressed in the alternative form

$$
p_{g o}=\frac{1}{4} \int_{D}(2 \nu+1) e^{i \nu \pi / 2}\left[h_{\nu}^{(2)}\left(k_{0} r\right)-\frac{h_{\nu}^{(2)^{\prime}}(K)}{h_{\nu}^{(1)^{\prime}}(K)} h_{\nu}^{(1)}\left(k_{0} r\right)\right] R_{\nu}^{0}(\cos \theta) d \nu
$$

where we have used $h_{\nu}^{(1)}$ and $h_{\nu}^{(2)}$ instead of $j_{\nu}$, and we wish to study this integral in the independent but simultaneous limits $K \rightarrow \infty$ and $k_{0} r \rightarrow \infty$. Following Nussenzveig [15], the first term (involving $h_{\nu}^{(2)}\left(k_{0} r\right)$ ) does not contribute at all at leading order and what remains is to obtain appropriate estimates of the second term, which is given by the approximate integral representation

$$
p_{g o} \sim-\left(\frac{1}{2 \pi \sin \theta}\right)^{\frac{1}{2}} e^{i \pi / 4-i \theta / 2} \int_{D} \nu^{1 / 2} \frac{h_{\nu}^{(2)^{\prime}}(K)}{h_{\nu}^{(1)^{\prime}}(K)} h_{\nu}^{(1)}\left(k_{0} r\right) e^{i \nu\left(\frac{\pi}{2}-\theta\right)} d \nu,
$$

in which $R_{\nu}^{0}(\cos \theta)$ appearing within the integrand in (31) has been approximated using (30) under the assumption that the principal contributions will come from large values of $\nu$. In broad terms, Debye-type asymptotic expansions, in which the order and argument of the Bessel functions are comparable (see [13]), are now used on the three spherical Bessel 
functions within the integrand in (32) and steepest descents methods applied; two principal contributions emerge, one being the incident field and the other the reflected field according as $\nu \sim K$ or $\nu \sim k_{0} r$, respectively.

More specifically, making the change of variable $\nu=\mu-\frac{1}{2}$ removes the exponential factor multiplying the integral and has the added advantage of leaving the integrand in terms of spherical Bessel functions of order $\mu-\frac{1}{2}$, which can then be expressed in terms of ordinary Bessel functions of order $\mu$ (and specifically not $\mu \pm \frac{1}{2}$ ). This allows standard asymptotic results to be quoted and used without any modifications being necessary to account for the half-integer that would otherwise have been present in the order (it is very tempting, for example, to say that $h_{\mu-\frac{1}{2}}^{(1,2)}(z) \sim h_{\mu}^{(1,2)}(z)$ as $\mu \rightarrow \infty$, but doing so will in general lead to the omission of $O(1)$ contributions to the phases of some terms within our integrand, especially when Debye-type limits are being taken).

The integral to be estimated is now

$$
p_{g o} \sim-\left(\frac{1}{2 \pi \sin \theta}\right)^{\frac{1}{2}} \int_{\mathrm{D}} \mu^{\frac{1}{2}} \frac{h_{\mu-\frac{1}{2}}^{(2)^{\prime}}(K)}{h_{\mu-\frac{1}{2}}^{(1)^{\prime}}(K)} h_{\mu-\frac{1}{2}}^{(1)}\left(k_{0} r\right) e^{i \mu\left(\frac{\pi}{2}-\theta\right)} d \mu .
$$

It is clear from (33) that two distinct ranges of $\mu$ are going to feature in the asymptotic structure of $p_{g o}$ :for large $K, k_{0} r$,

$$
\begin{aligned}
\text { (i) } \mu-\frac{1}{2} & =O(K), \\
\text { (ii) } \mu-\frac{1}{2} & =O\left(k_{0} r\right),
\end{aligned}
$$

these being the values of $\nu$ for which the order and argument of each of the two different types of spherical Bessel function that arise are of similar sizes, necessitating the use of Debye-type expansions. Notice that just as in the problem considered in [15], both of our arguments $\left(K\right.$ and $k_{0} r$ ) are large, but our situation is quite different in that $k_{0} r$ is much larger than $K$, and so we will encounter situations where we use Debye expansions on one part of the integrand but apply a standard leading-order asymptotic expansion on the other - something that never occurred in [15].

In fact, exactly this happens in case (i) for which $\mu-\frac{1}{2}=O(K)$, when the term $h_{\mu-\frac{1}{2}}^{(1)}\left(k_{0} r\right)$ has its argument greatly exceeding its order, in direct contrast with the ratio of spherical Bessel functions for which the order and argument are comparable. Since

$$
\frac{h_{\mu-\frac{1}{2}}^{(2)^{\prime}}(K)}{h_{\mu-\frac{1}{2}}^{(1)^{\prime}}(K)} \sim-e^{-2 i\left[\sqrt{K^{2}-\mu^{2}}-\mu \cos ^{-1}\left(\frac{\mu}{K}\right)-\frac{\pi}{4}\right]}
$$


as $K \rightarrow \infty$ for $\mu-\frac{1}{2}=O(K)$, it now follows that the contribution $p_{g o}^{(1)}$ to $p_{g o}$ from this region of the integration path is given by

$$
p_{g o}^{(1)} \sim\left(\frac{1}{2 \pi \sin \theta}\right)^{\frac{1}{2}} e^{i \pi / 4} \frac{e^{i k_{0} r}}{k_{0} r} \int_{-\infty}^{\infty} \mu^{\frac{1}{2}} e^{i\left[-2 \sqrt{K^{2}-\mu^{2}}+2 \mu \cos ^{-1}\left(\frac{\mu}{K}\right)-\mu \theta\right]} d \mu,
$$

to which a standard stationary phase argument can be applied to yield

$$
p_{g o}^{(1)} \sim \frac{a b \epsilon}{2(b-a)} e^{-2 i K \sin \left(\frac{1}{2} \theta\right)} \frac{e^{i k_{0} r}}{r} .
$$

This is a scattered wave radiating away from $r=b$ with spherical wave fronts concentric with the cloak, and can be identified as a reflected wave of $O(\epsilon)$ amplitude.

The other principal contribution (case (ii)) is such that the functions $h_{\mu-\frac{1}{2}}^{(1)^{\prime}}(K), h_{\mu-\frac{1}{2}}^{(2)^{\prime}}(K)$ now have orders significantly exceeding their arguments, and then

$$
\frac{h_{\mu-\frac{1}{2}}^{(2)^{\prime}}(K)}{h_{\mu-\frac{1}{2}}^{(1)^{\prime}}(K)} \sim-1
$$

A Debye expansion is now necessary for the spherical Bessel function dependent upon $k_{0} r$ and the approximation

$$
p_{\text {go }}^{(2)} \sim e^{-i \pi / 4}\left(\frac{k_{0} r}{2 \pi \sin \theta}\right)^{\frac{1}{2}} \int_{-\infty}^{\infty} \frac{\lambda^{\frac{1}{2}}}{\left(1-\lambda^{2}\right)^{\frac{1}{4}}} e^{i k_{0} r\left[\sqrt{1-\lambda^{2}}-\lambda \cos ^{-1}(\lambda)+\lambda\left(\frac{\pi}{2}-\theta\right)\right]} d \lambda
$$

results, which can be further simplified to yield

$$
p_{\text {go }}^{(2)} \sim e^{i k_{0} r \cos \theta},
$$

and this contribution therefore supplies the incident wave.

\subsection{Cylindrical cloaks}

We now consider the regularised cloaking problem with Neumann data prescribed at the target located at $r=a(1+\epsilon)$, and with outer cloak radius $r=b$. Assuming a standard incident plane wave $p_{i n c}=e^{i k_{0} r \cos \theta}$ in cylindrical polar coordinates, we find that the total field can be written as

$$
p=p_{0} e^{i k_{0} R(r) \cos \theta}-p_{0} \sum_{n=0}^{\infty} i^{n}\left(2-\delta_{n, 0}\right) \frac{J_{n}^{\prime}\left(k_{0} R(a(1+\epsilon))\right)}{H_{n}^{(1)^{\prime}}\left(k_{0} R(a(1+\epsilon))\right)} H_{n}^{(1)}\left(k_{0} R(r)\right) \cos (n \theta),
$$

where $J_{n}, H_{n}^{(1)}$ are cylindrical Bessel and Hankel functions and

$$
R(r)= \begin{cases}b(r-a) /(b-a) & a(1+\epsilon) \leq r<b \\ r & b \leq r<\infty\end{cases}
$$


Alternatively, with an eye towards obtaining an integral solution whose integrand is analogous to that in (31) for the spherical case, so that hopefully some of the asymptotic results used there carry over to this situation with minimal effort, we re-express (40) to give a modified version of the total field in $r>b$ in the particular form

$$
p_{t}(r, \theta)=\frac{1}{2} \sum_{n=-\infty}^{\infty} i^{n}\left[H_{n}^{(2)}\left(k_{0} r\right)-\frac{H_{n}^{(2)^{\prime}}(K)}{H_{n}^{(1)^{\prime}}(K)} H_{n}^{(1)}\left(k_{0} r\right)\right] e^{-i n \theta} .
$$

We can immediately deduce from this that the relevant function $f(\nu)$ in (15) is given by

$$
f(\nu)=-\frac{i}{4}\left[H_{\nu}^{(2)}\left(k_{0} r\right)-\frac{H_{\nu}^{(2)^{\prime}}(K)}{H_{\nu}^{(1)^{\prime}}(K)} H_{\nu}^{(1)}\left(k_{0} r\right)\right] e^{-i \nu\left(\theta+\frac{\pi}{2}\right)},
$$

so that our integral solution now becomes

$$
p_{t}(r, \theta)=-\frac{i}{4} \int_{D}\left[H_{\nu}^{(2)}\left(k_{0} r\right)-\frac{H_{\nu}^{(2)^{\prime}}(K)}{H_{\nu}^{(1)^{\prime}}(K)} H_{\nu}^{(1)}\left(k_{0} r\right)\right] e^{-i \nu\left(\theta+\frac{\pi}{2}\right)} \frac{d \nu}{\sin (\nu \pi)} .
$$

Broadly speaking, when evaluating (44) as both $k_{0} r \rightarrow \infty$ and $K \rightarrow \infty$, the ideas from the analysis of the spherical geometry carry over almost directly, so that the first term within the integrand (proportional to $H_{\nu}^{(2)}\left(k_{0} r\right)$ ) does not contribute at all. In what remains, there are (i) the residue contributions from the zeroes of $\sin (\nu \pi)$, which get transferred via a deformation of contours into those from the zeroes of $H_{\nu}^{(1)^{\prime}}(K)$ (thus providing a rapidly convergent series contribution), (ii) those contributions from the integration range when $\nu=O\left(k_{0} r\right)$, when the ratio $\frac{H_{\nu}^{(2)^{\prime}}(K)}{H_{\nu}^{(1)^{\prime}}(K)}$ is both constant and independent of $a$ and $\epsilon$ and finally (iii) contributions from $\nu=O(K)$ when the sensitivity of the afore-mentioned ratio on $a$ and $\epsilon$ is paramount, whilst the third Hankel function $\left(H_{\nu}^{(1)}\left(k_{0} r\right)\right)$ then just represents an outgoing cylindrically-spreading wave of principal phase $k_{0} r$ and with an amplitude proportional to $\left(k_{0} r\right)^{-1 / 2}$. The terms in (i) are the diffracted field, those in (ii) combine to yield the incident field (which is why that element is independent of both $a$ and $\epsilon$ - the incident field is a stand-alone contribution and is always an exact solution of the Helmholtz equation completely independent of the presence or otherwise of any obstructing boundary) and those in (iii) are the ones in which we are most interested since they give the 'geometrical optics' specularly reflected field.

Restricting attention to case (iii), we replace $H_{\nu}^{(1)}\left(k_{0} r\right)$ by its standard asymptotic expansion (since exterior to the cloak $k_{0} r \gg K \gg 1$ ), and this includes a multiplicative factor $e^{-i \nu \pi / 2}$. This combines with another such factor already present within the integrand of (44) to give an overall factor $e^{-i \nu \pi}$, which we then write as

$$
e^{-i \nu \pi}=-2 i \sin (\nu \pi)+e^{i \nu \pi} .
$$

The second term on the right-hand side of this decomposition maintains the $\sin (\nu \pi)$ term on the denominator of (44) and becomes a part of the total diffracted field referred to just 
now as case (i). However, the first term on the same right-hand side removes this $\sin (\nu \pi)$ term altogether, leaving behind an asymptotic representation of the reflected field $p_{r}$ in the integral form

$$
p_{r}(r, \theta) \sim-\frac{i}{\sqrt{2 \pi}} \frac{e^{i k_{0} r+i \pi / 4}}{\sqrt{k_{0} r}} \int_{-\infty}^{\infty} \frac{H_{\nu}^{(2)^{\prime}}(K)}{H_{\nu}^{(1)^{\prime}}(K)} e^{-i \nu \theta} d \nu
$$

evaluation of which as $K \rightarrow \infty$ is by now elementary (using the Debye expansions of the ratio of differentiated Hankel functions) and is given by

$$
p_{\text {go }}(r, \theta) \sim \sqrt{\frac{a b \epsilon}{b-a}} \sqrt{\frac{1}{2 r} \sin \left(\frac{1}{2} \theta\right)} e^{i k_{0} r-2 i K \sin \left(\frac{1}{2} \theta\right)} .
$$

Note in particular that on $r=b$,

$$
p_{g o}(b, \theta) \sim \sqrt{\frac{a \epsilon}{2(b-a)} \sin \left(\frac{1}{2} \theta\right)} e^{i k_{0} b-2 i K \sin \left(\frac{1}{2} \theta\right)},
$$

- a result to which we shall refer in Section 3.2.

\section{Ray theory}

\subsection{Spherical targets}

Ray theory has been applied to the numerical solution of cloaking models by several authors (Gao et al [12], Tanaka et al, [16], Crosskey et al [17] and Hu et al [18], for example). Here we will use the method to obtain explicit asymptotic solutions for large $K .^{3}$

\subsubsection{Target $r=a$}

For large values of $k_{s h}$ and a spherical target $r=a$, the WKB expansion is

$$
p \sim e^{i k_{s h} u}\left(A_{0}+\frac{1}{k_{s h}} A_{1}+\ldots\right) .
$$

We note immediately that if we follow the ray starting at $r=b$ and $\theta=\theta_{0}$, and use the fact that the rays in the TO plane given by (13) are straight lines parallel to the $x$-axis, then the incoming ray is given by

$$
r-a=(b-a) \frac{\sin \theta_{0}}{\sin \theta}
$$

and the associated phase and amplitude are given by $u=(r-a) \cos \theta$ and $A_{0}=1$. Hence we have arrived at the exact solution stated in (6) and we find $A_{i}=0$ for $i \geq 1$ in (48).

The ray geometry is shown in Figure 2 which gives a geometric interpretation of the way in which the incident wave energy in $r|\sin \theta|<b$ is diverted around the target. This situation changes when the target is at $r=a(1+\epsilon)$.

\footnotetext{
${ }^{3}$ In practice the wavelength must always be larger than that of the microstructure of the cloaking material.
} 


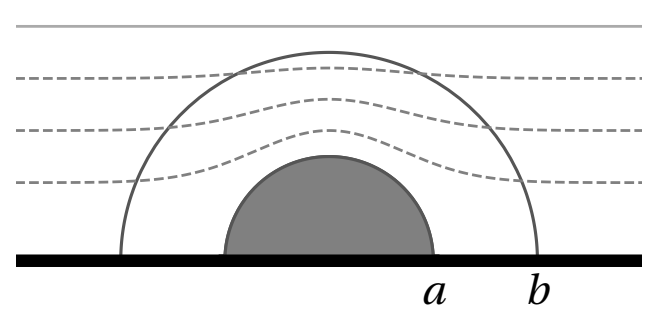

Figure 2: Rays in a perfect cloak

\subsubsection{Target $r=a(1+\epsilon)$}

Expanding the target to $r=a(1+\epsilon)$ in order to keep the density and bulk modulus finite cannot affect the incident field described above. Also, it is clear from Fig. 3 that only the rays for which $\pi-\theta_{0}=O(\epsilon)$ can impact the target, the limiting ray being given from (49) by

$$
\theta_{0}=\pi-\sin ^{-1}\left(\frac{a \epsilon}{b-a}\right)
$$

where here, and henceforth, the inverse sine is taken to lie in $\left(-\frac{\pi}{2}, \frac{\pi}{2}\right)$. Hence we are led to consider a boundary layer in which

$$
\tilde{r}=\frac{r-a}{\epsilon a}=O(1)
$$

and $p$ satisfies the classical Helmholtz equation

$$
\tilde{r}^{2} \frac{\partial^{2} p}{\partial \tilde{r}^{2}}+2 \tilde{r} \frac{\partial p}{\partial \tilde{r}}+\frac{1}{\sin \theta} \frac{\partial}{\partial \theta}\left(\sin \theta \frac{\partial p}{\partial \theta}\right)+K^{2} \tilde{r}^{2} p=0
$$

where $K$ must be large for ray theory to apply. In contrast to Section 2, we first assume the Dirichlet condition $p=0$ on $\tilde{r}=1$ and, when we subtract out the known incident field, the boundary condition for the specularly reflected field is

$$
p_{s c}=-e^{i K \cos \theta} \quad \text { on } \quad \tilde{r}=1 .
$$

Since (52) is (1) with $r-a$ replaced by $\tilde{r}$ and $k_{s h}$ replaced by $K$, the ray model described in the previous case also applies to the specularly reflected field of rays formed when the incident rays impact the boundary. For an incident ray parametrised by $\theta_{0}$, this impact happens at

$$
\theta=\theta_{1}=\pi-\sin ^{-1}\left(\frac{(b-a) \sin \theta_{0}}{a \epsilon}\right) .
$$

Writing $p_{s c} \sim A_{s c} e^{i K \tilde{u}}$, the eikonal equation for (52) is

$$
\left(\frac{\partial \tilde{u}}{\partial \tilde{r}}\right)^{2}+\frac{1}{\tilde{r}^{2}}\left(\frac{\partial \tilde{u}}{\partial \theta}\right)^{2}=1
$$


and the associated Charpit equations (Ockendon et al [19]) are

$$
\frac{d \tilde{r}}{d t}=2 \frac{\partial \tilde{u}}{\partial \tilde{r}}, \frac{d \theta}{d t}=\frac{2}{\tilde{r}^{2}} \frac{\partial \tilde{u}}{\partial \theta}, \frac{d \tilde{u}}{d t}=2, \frac{d}{d t}\left(\frac{\partial \tilde{u}}{\partial \tilde{r}}\right)=\frac{2}{\tilde{r}^{3}}\left(\frac{\partial \tilde{u}}{\partial \theta}\right)^{2}, \frac{d}{d t}\left(\frac{\partial \tilde{u}}{\partial \theta}\right)=0,
$$

which must be solved subject to

$$
\tilde{r}=1, \tilde{u}=\cos \theta_{1}, \frac{\partial \tilde{u}}{\partial \theta}=-\sin \theta_{1}, \frac{\partial \tilde{u}}{\partial \tilde{r}}=-\cos \theta_{1} \quad \text { when } \quad \theta=\theta_{1} .
$$

After some manipulation we find that the specularly reflected rays are

$$
\tilde{r}=-\frac{\sin \theta_{1}}{\sin \left(\theta-2 \theta_{1}\right)} \quad \text { with } \quad \tilde{u}=2 \cos \theta_{1}+\sin \theta_{1} \cot \left(\theta-2 \theta_{1}\right)
$$

and hence that these rays leave the boundary layer at $\theta=\theta_{2}=2 \theta_{1}-\pi$, leading to the ray pattern in Figure 3. We note that this result could also have been obtained by mapping into the TO plane since specular reflection is preserved.

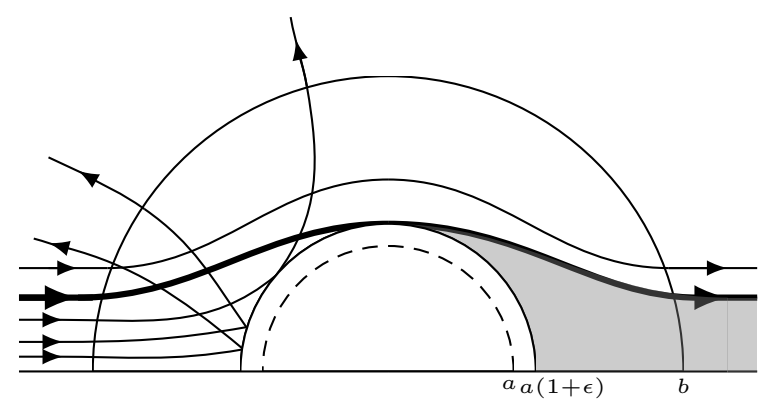

Figure 3: Rays in an imperfect cloak. The shadow region is shown shaded.

This scattered field reduces to that for classical scattering as $\epsilon$ increases and the cloak thickness vanishes. However, our WKB solution is not uniformly valid as $\epsilon \rightarrow 0$ as we now discuss.

We begin by noting that the incoming rays on which $\theta_{0}=O(\epsilon)$ are almost rectangular hyperbolae in a region where $\tilde{r}=O\left(\epsilon^{-1 / 2}\right), \pi-\theta=O\left(\epsilon^{1 / 2}\right)$ and the lowest order ray theory solution is still the incident wave $p=e^{-i K \tilde{r} \cos \theta}$. However, we need to go to smaller values of $\tilde{r}-1$ and $\pi-\theta$ before we encounter a scattered field that is not described by ray theory. We notice that if $\tilde{r}-1 \sim \pi-\theta \sim O(\delta),\left(\delta \ll \epsilon^{1 / 2}\right)$, our ray theory remains valid as long as $K \delta \gg 1$. But, when $\delta \sim \frac{1}{K},(52)$ can be rescaled to become a Helmholtz equation with coefficients of $O(1)^{4}$.

\footnotetext{
${ }^{4}$ Such problems arise in all scattering problems for blunt targets and, in this case, the model will predict a scattered field near $\theta=\pi$ which is independent of $\epsilon$ and which decays with a suitable power of distance from the focus of the osculating parabola or paraboloid at the point of minimum curvature. However, this scenario only holds when $K \epsilon^{1 / 2}$ is small; when this parameter is of $O(1)$, the solution of the relevant Helmholtz equation will need to match to a far-field that is non-radial, which will result in a different scattered field near $\theta=\pi$.
} 
There is another non-uniformity near $\tilde{r}=1, \theta=\frac{\pi}{2}$, where the incident and reflected rays graze the target. Such grazing phenomena in homogeneous media have been extensively studied (see, for example, Ockendon and Tew [20]), and they typically lead to an inner region described by a version of the parabolic wave equation known as the Fock-Leontovich equation. Solutions of this equation match with an incident grazing plane wave and a transmitted shadow boundary near the outgoing grazing ray (Fig. 3). Between this shadow boundary and the target, a deep shadow region is formed in which there are real 'creeping rays' with exponentially small amplitude and a similar scenario is expected to apply to our problem even though the grazing rays are now curved.

Our final and most important calculation is that of the asymptotic form of the reflected wavefield which can only be determined by a more complicated calculation involving the transport equation for the reflected wavefield as $\theta \rightarrow \theta_{2}$. The lowest order amplitude $A_{s c}$ satisfies

$$
P \frac{\partial A_{s c}}{\partial \tilde{r}}+Q \frac{\partial A_{s c}}{\partial \theta}+S A_{s c}=0
$$

with $A_{s c}=-1$ at $\theta=\theta_{1}$, where

$$
P=2 \tilde{r}^{2} \frac{\partial \tilde{u}}{\partial \tilde{r}}, Q=2 \frac{\partial \tilde{u}}{\partial \theta} \quad \text { and } \quad S=\tilde{r}^{2} \frac{\partial^{2} \tilde{u}}{\partial \tilde{r}^{2}}+2 \tilde{r} \frac{\partial \tilde{u}}{\partial \tilde{r}}+\frac{\partial^{2} \tilde{u}}{\partial \theta^{2}}+\cot \theta \frac{\partial \tilde{u}}{\partial \theta} .
$$

Along a ray, this eventually yields the equation

$$
\frac{1}{A_{s c}} \frac{d A_{s c}}{d \theta}=\frac{S}{2 \sin \theta_{1}}
$$

where

$$
S=\frac{2 \sin ^{2} \theta_{1}-\sin \theta_{1} \cos \theta_{1} \cot \left(\theta-2 \theta_{1}\right)}{2 \sin \theta_{1} \cot \left(\theta-2 \theta_{1}\right)+\cos \theta_{1}}+2 \sin \theta_{1} \cot \left(\theta-2 \theta_{1}\right)-\cot \theta \sin \theta_{1}
$$

this in turn yields

$$
A_{s c}=\sin \left(\theta-2 \theta_{1}\right) \sqrt{\frac{\cos \theta_{1}}{\sin \theta\left(\cos \theta_{1} \sin \left(\theta-2 \theta_{1}\right)+2 \sin \theta_{1} \cos \left(\theta-2 \theta_{1}\right)\right)}} .
$$

Thus, as $\theta \rightarrow \theta_{2}=2 \theta_{1}-\pi, \tilde{r}=\frac{\sin \theta_{1}}{\sin \left(\theta-\theta_{2}\right)}$ and so $A_{s c} \sim-\frac{1}{2 \tilde{r}}$ and hence

$$
p_{s c} \sim-\frac{1}{2 \tilde{r}} e^{i K\left(\tilde{r}-2 \sin \left(\frac{1}{2} \theta\right)\right)} \quad \text { as } \quad \tilde{r} \rightarrow \infty .
$$

Since the scattered field satisfies the same equation throughout the cloak, the scattered field at $r=b$ is $-\frac{\epsilon a}{2(b-a)} e^{i k_{s h}(b-a)-2 i K \sin \left(\frac{1}{2} \theta\right)}$, which can be written in the equivalent form

$$
-\frac{a b \epsilon}{2(b-a)} e^{-2 i K \sin \left(\frac{1}{2} \theta\right)} \frac{e^{i k_{0} b}}{b}
$$


Hence the scattered far field in $r>b$ is of the spherically-spreading form $d(\theta) \frac{e^{i k_{0} r}}{r}$, where $d(\theta)=-\frac{\epsilon a b}{2(b-a)} e^{-2 i K\left(\sin \frac{1}{2} \theta\right)}$, with an amplitude which is both radially symmetric and of $O(\epsilon)$, uniformly as $r \rightarrow \infty$. We note that in the WKB approximation, the Neumann problem only differs from the Dirichlet problem in that the sign of $p_{s c}$ is changed and hence this result is in complete agreement with the solution (36) obtained in Section 2.1.2.

\subsection{Cylindrical Cloaks}

For a cylindrical target in which $(r, \theta)$ are cylindrical rather than spherical polar coordinates, the only modification to (1) is the removal of the coefficient 2 in the second term and the replacement of the third term by $\frac{\partial^{2} p}{\partial \theta^{2}}$.

When the target is at $r=a$, the calculations for the phase and rays are exactly as in the spherical case in Section 3.1.1, and again ray theory provides the exact solution to the full problem. The same remarks apply when the cylinder is perturbed to $r=a(1+\epsilon)$, with the sole exception that $A_{s c}$ is no longer given by (61). It turns out that

$$
\frac{1}{A_{s c}} \frac{d A_{s c}}{d \theta}=\frac{S}{2 \sin \theta_{1}}
$$

as before, but now, instead of (60),

$$
S=\frac{2 \sin \theta_{1}}{\left(\cot \theta_{1}+2 \cot \left(\theta-2 \theta_{1}\right)\right) \sin ^{2}\left(\theta-2 \theta_{1}\right)},
$$

with $\theta_{1}(\tilde{r}, \theta)$ still given by (58). Thus, since $A_{s c}=-1$ at $\theta=\theta_{1}$,

$$
A_{s c}=-\sqrt{-\frac{\cot \theta_{1}}{\cot \theta_{1}+2 \cot \left(\theta-2 \theta_{1}\right)}}
$$

The scattered amplitude as $\theta \rightarrow 2 \theta_{1}-\pi$ and $\tilde{r}=-\frac{\sin \theta_{1}}{\sin \left(\theta-2 \theta_{1}\right)} \rightarrow \infty$ is given by

$$
A_{s c} \sim-\sqrt{-\frac{\cos \theta_{1}}{2 \tilde{r}}}=-\sqrt{\frac{\sin \left(\frac{1}{2} \theta\right)}{2 \tilde{r}}}
$$

Not only does the field have the expected inverse square root behaviour in $\tilde{r}$, but its ampli-

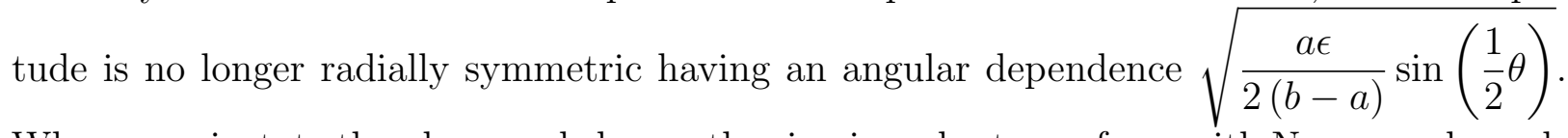
When we reinstate the phase and change the sign in order to conform with Neumann boundary conditions this is in perfect agreement with (47) in Section 2.2. 


\subsection{Thin cloaks}

We conclude this Section with a brief discussion of how cloaking deteriorates for thin cloaks. When we formally take the limit $\frac{b}{a} \downarrow 1$ in the model (52), we retrieve the classical problem of scattering by a sphere or a cylinder in a homogeneous medium. Since the scattered field is dramatically reduced by the cloak when $\frac{b}{a}=O(1)$ and the target is $r=a(1+\epsilon)$, we now consider the situation in which $b=a(1+\gamma \epsilon)$ with $\gamma>1$. Again we will restrict considerations to the case $K \gg 1$ and we begin with the spherical case.

Away from the areas of non-uniformity mentioned in Section 3.1.2, the phase of the incoming wave field in the cloak is still

$$
u=\tilde{r} \cos \theta
$$

with the incident rays being

$$
\tilde{r}=\gamma \frac{\sin \theta_{0}}{\sin \theta}
$$

Thus, writing $\theta_{0}^{*}=\pi-\sin ^{-1}(1 / \gamma)$ with $\frac{\pi}{2}<\theta_{0}^{*}<\pi$, we see that the rays for which $\theta_{0}>\theta_{0}^{*}$ will reflect from the target. As $\gamma \rightarrow \infty$, a smaller and smaller proportion of incident rays will impact the target and from (61), on the cloak boundary, $2 \theta_{1}-\theta=\theta_{0}$ and $\theta_{0} \rightarrow \pi$ so that

$$
A_{s c} \sim-\frac{\sin \theta_{0}}{2 \sin \theta_{1}}
$$

Hence, we see that $A_{s c} \sim-1 /(2 \gamma)$ as $\gamma \rightarrow \infty$.

In the cylindrical case the corresponding result is, from (62), that $A_{s c} \sim-\sqrt{\frac{\sin \left(\frac{1}{2} \theta\right)}{2 \gamma}}$. Note that both these results are in line with those of Sections 3.1 .2 and 3.2 when $\gamma=O\left(\frac{1}{\epsilon}\right)$.

\section{Generalised models}

\subsection{Point source insonification}

We now consider a spherical target $r=a$ whose cloak is modelled by (1) but which is now insonified by a source situated at $(r, \theta, \phi)=(d, 0,0)$ in $d>b$ given by

$$
p=\frac{e^{i k_{0} R}}{4 \pi R}=\frac{i k_{0}}{4 \pi} \sum_{n=0}^{\infty}(2 n+1) h_{n}^{(1)}\left(k_{0} d\right) j_{n}\left(k_{0} r\right) P_{n}(\cos \theta), \quad R<d,
$$

where

$$
R=\left(r^{2}+d^{2}-2 r d \cos \theta\right)^{1 / 2}
$$


Then we find the field in the cloak is given by

$$
p=\sum_{n=0}^{\infty} B_{n} j_{n}\left(k_{s h}(r-a)\right) P_{n}(\cos \theta)
$$

where

$$
B_{n}=\frac{i k_{0}}{4 \pi}(2 n+1) h_{n}^{(1)}\left(k_{0} d\right) b^{-1 / 2}(b-a)^{-1 / 2}
$$

and so

$$
p=\frac{i k_{0}}{4 \pi} \sum_{n=0}^{\infty}(2 n+1) h_{n}^{(1)}\left(k_{0} d\right) j_{n}\left(k_{0} b \frac{(r-a)}{(b-a)}\right) P_{n}(\cos \theta) .
$$

Hence

$$
p=\frac{e^{i k_{0} R_{1}}}{4 \pi R_{1}} \quad \text { in } \quad a<r<b
$$

where

$$
R_{1}=\left(b^{2} \frac{(r-a)^{2}}{(b-a)^{2}}+d^{2}-2 b \frac{(r-a)}{(b-a)} d \cos \theta\right)^{1 / 2}, \quad b>r>a .
$$

and $R_{1}$ is a measure of distance from the image point $\left(\frac{d(b-a)}{b}+a, 00\right)$. Thus, for large values of $d$, the scattered field is of $O\left(d^{-1}\right)$ and it is easy to show that it would be of $O\left(d^{-1 / 2}\right)$ in the cylindrical case. However, all cloaking would be lost if sources were distributed uniformly on $r=d$ so as to produce a radial incident wave.

We remark that the transformation optics technique in which the radial distance $r$ is replaced by $(r-a)$ can also be used to

(i) solve the problem of a homogeneous spherical target with a point source embedded at $r=0$ and(ii) analyse the effect of a composite cloak in $c<r<b$, in which, say,

$$
\rho=\frac{a-c}{c} \frac{r^{2}}{(r-a)^{2}} \quad \text { for } \quad c<r<a \quad \text { with } \quad c=\frac{a b}{2 b-a},
$$

in addition to (4).

\subsection{Material properties proportional to $(r-a)^{\alpha}, 2>\alpha>0$}

In anticipation that solutions of (52) will have radically different properties when the $\tilde{r}^{-}$ dependent coefficients are replaced by constants, we now consider the effect of moderating the singularities in $\lambda$ and $\underline{\boldsymbol{\rho}}$ as $r \rightarrow a$. For simplicity, we will restrict attention to the configurations with $\left(\frac{b}{a}-1\right)=O(1)$.

We replace (7) by

$$
\lambda=\frac{(b-a)^{\alpha+1} r^{2}}{b^{3}(r-a)^{\alpha}} \quad \text { for } \quad a<r<b,
$$


for $0<\alpha<2$, so that the singularity at $r=a$ is less severe than was the case in the previous Sections.

Correspondingly, we choose the anisotropic density

$$
\underline{\underline{\rho}}=\left(\begin{array}{ccc}
\frac{(b-a)^{\alpha-1} r^{2}}{b(r-a)^{\alpha}} & 0 & 0 \\
0 & \frac{b-a}{b} & 0 \\
0 & 0 & \frac{b-a}{b}
\end{array}\right) .
$$

Substitution of (68) and (69) into (11) reveals that (1) is replaced by

$$
(r-a)^{\alpha} \frac{\partial^{2} p}{\partial r^{2}}+\alpha(r-a)^{\alpha-1} \frac{\partial p}{\partial r}+\frac{(b-a)^{\alpha-2}}{\sin \theta} \frac{\partial}{\partial \theta}\left(\sin \theta \frac{\partial p}{\partial \theta}\right)+k_{s h}^{2}(r-a)^{\alpha} p=0 .
$$

We will only consider the high-frequency limit in which $k_{s h} \rightarrow \infty$ and then the phase $u$ in (48) satisfies

$$
\left(\frac{\partial u}{\partial r}\right)^{2}+\frac{(b-a)^{\alpha-2}}{(r-a)^{\alpha}}\left(\frac{\partial u}{\partial \theta}\right)^{2}=1
$$

With the usual incident field $p=e^{i k_{0} r \cos \theta}$, the boundary conditions on $r=b$ are still given by (??), but Charpit's equations are now

$$
\begin{gathered}
\frac{d r}{d t}=2 \frac{\partial u}{\partial r}, \quad \frac{d \theta}{d t}=\frac{2(b-a)^{\alpha-2}}{(r-a)^{\alpha}} \frac{\partial u}{\partial \theta}, \quad \frac{d u}{d t}=2 \\
\frac{d}{d t}\left(\frac{\partial u}{\partial r}\right)=\frac{2(b-a)^{\alpha-2}}{(r-a)^{\alpha+1}}\left(\frac{\partial u}{\partial \theta}\right)^{2} \quad \text { and } \quad \frac{d}{d t}\left(\frac{\partial u}{\partial \theta}\right)=0 .
\end{gathered}
$$

When $\theta_{0}=\pi, \frac{\partial u}{\partial \theta}=0$ and so $\theta=\pi$ and this ray will reach the target $r=a$. However, when $\theta_{0}<\pi$, the differential equation for the ray through $r=b, \theta=\theta_{0}$ is

$$
\frac{d r}{d \theta}=\frac{\left[(r-a)^{\alpha}\left((r-a)^{\alpha}\left(\cos ^{2} \theta_{0}+\frac{2}{\alpha} \sin ^{2} \theta_{0}\right)-\frac{2}{\alpha}(b-a)^{\alpha} \sin ^{2} \theta_{0}\right)\right]^{1 / 2}}{(b-a)^{\alpha-1} \sin \theta_{0}},
$$

where the positive value of the square root is taken since $r$ and $\theta$ are both decreasing on the incoming ray. Hence we see that $r$ will have a minimum when

$$
r-a=(b-a)\left[\frac{2 \sin ^{2} \theta_{0}}{\alpha \cos ^{2} \theta_{0}+2 \sin ^{2} \theta_{0}}\right]^{1 / \alpha},
$$

which will always occur in $r>a$ for $0<\alpha<2$. Once this minimum value has been achieved, $r$ will increase while $\theta$ continues to decrease and so it is necessary to take the negative square root in (74) on this latter part of the ray.

It can further be shown that as $\alpha$ decreases from 2 the value of $\theta$ for which the ray entering the cloak when $\theta=\theta_{0}$ refracts into the homogeneous medium at $r=b$ will increase from $\pi-\theta_{0}$. Thus we see that the effect of the cloak weakens as $\alpha$ decreases. 


\subsection{Low-frequency cloaking}

Hitherto, we have not considered cloaking at frequencies low enough for $k_{0}$ and $k_{s h}$ to be of $O(1)$ or smaller. When these parameters are of $O(1)$ and the target is a sphere $r=a(1+\epsilon)$, we can write down expansions for $p$ in powers of $\epsilon$, with (6) being the lowest order solution except in a boundary layer where $\tilde{r}=O(1)$. In this layer, $p$ will be a harmonic function of $(\tilde{r}, \theta)$ to lowest order, whose solution is $p=1-\frac{1}{\tilde{r}}$ for zero Dirichlet data on $\tilde{r}=1$. The scattered field in the cloak will then satisfy the Helmholtz equation (52) with $p \sim-\frac{1}{r-a}$ as $r \downarrow a$, while, for Neumann data, we would have to go to higher orders in $\epsilon$. We will not pursue this regime further here except to note that the scattered field will be of $O(\epsilon)$ for Dirichlet data and $o(\epsilon)$ for Neumann data and that the corresponding cylindrical problem will involve expansions in powers of $\frac{1}{(\log \epsilon)}$. A general discussion of low-frequency scattering is given by Dassios and Kleinman [22] and we will simply record the lowest-order fields when $k_{0}$ and $k_{s h}$ are small. In this case, explicit asymptotic expansions for $p$ can be found by perturbing about the static solution when $k_{0}=k_{s h}=0$. For the canonical regime in which $k_{0}$ and $k_{s h}$ are of $O(\epsilon)$, the leading terms are as follows, where $\mathrm{D}$ and $\mathrm{N}$ denote Dirichlet and Neumann conditions, respectively. In each case, the same four spatial regions need to be considered asymptotically and these regions are defined in the first case.

a) Spherical Target, $r=a(1+\epsilon)$

$$
\begin{array}{rlrl}
\mathrm{D}: & 1 & r & \gtrsim O\left(\frac{1}{\epsilon}\right): \quad p \sim e^{i k_{0} r \cos \theta}-\frac{\epsilon a b}{r(b-a)} e^{-i k_{0} r}+O\left(\epsilon^{2}\right) . \\
2 & \frac{1}{\epsilon} \gg r>b: \quad p \sim 1+i k_{0} r \cos \theta-\frac{\epsilon a b}{r(b-a)}+O\left(\epsilon^{2}\right) . \\
3 & b-a>r-a \gg a \epsilon: \quad p \sim 1-\frac{\epsilon a}{r-a}+i k_{s h}(r-a) \cos \theta+O\left(\epsilon^{2}\right) . \\
& & \tilde{r} & =\frac{r-a}{a \epsilon}=O(1): \quad p \sim 1-\frac{1}{\tilde{r}}+i \epsilon k_{s h} \cos \theta\left(\tilde{r}-\frac{1}{\tilde{r}^{2}}\right)+O\left(\epsilon^{2}\right) . \\
\mathrm{N}: \quad & p & \sim e^{i k_{0} r \cos \theta}+O\left(\epsilon^{3}\right) . \\
1 & p & \sim 1+i k_{0} r \cos \theta+O\left(\epsilon^{2}\right) . \\
2 & p & \sim 1+i k_{s h}(r-a) \cos \theta+O\left(\epsilon^{4}\right) . \\
3 & p & \sim 1+i \epsilon a k_{s h} \cos \theta\left(\tilde{r}+\frac{1}{2 \tilde{r}^{2}}\right)+O\left(\epsilon^{4}\right) .
\end{array}
$$


b) Cylindrical Target, $r=a(1+\epsilon)$

$$
\begin{array}{rlrl}
\mathrm{D}: & 1 & p & \sim e^{i k_{0} r \cos \theta}-\frac{\pi}{2 i} \frac{h_{0}^{(1)}\left(k_{0} r\right)}{\log \epsilon}+O(\epsilon) . \\
2 & & p \sim 1-\frac{\log (r(b-a) / a b)}{\log \epsilon}+i k_{0} r \cos \theta+O\left(\frac{\epsilon}{\log \epsilon}\right) . \\
3 & & \sim 1-\frac{\log ((r-a) / a)}{\log \epsilon}+i k_{s h}(r-a) \cos \theta+O\left(\frac{\epsilon}{\log \epsilon}\right) . \\
& & p & \sim-\frac{\log \tilde{r}}{\log \epsilon}+i \epsilon k_{s h} a\left(\tilde{r}-\frac{1}{\tilde{r}}\right) \cos \theta+O\left(\frac{\epsilon}{\log \epsilon}\right) . \\
\mathrm{N}: & 1 & p \sim e^{i k_{0} r \cos \theta}+\frac{i k_{0} \epsilon^{2} a^{2} b^{2}}{(b-a)^{2}} \frac{\cos \theta}{r}+O\left(\epsilon^{4}\right) . \\
2 & p \sim 1+i k_{0} r \cos \theta+\frac{i k_{0} \epsilon^{2} a^{2} b^{2}}{(b-a)^{2}} \frac{\cos \theta}{r}+O\left(\epsilon^{4}\right) . \\
3 & p \sim i k_{s h}(r-a) \cos \theta+O\left(\epsilon^{3}\right) . \\
4 & p \sim 1+i k_{s h} \epsilon a \cos \theta\left(\tilde{r}+\frac{1}{\tilde{r}}\right)+O\left(\epsilon^{3}\right) .
\end{array}
$$

We note that the above results for large $r$ under Dirichlet boundary conditions are consistent with the results in [4], [5].

As expected, the Neumann boundary condition produces much less scatter than the Dirichlet condition, as well as having different directionality.

\section{Conclusion}

This brief study of models for the cloaking of a special class of elastic spherical and cylindrical targets under arbitrary plane wave insonification has revealed that a class of cloaks whose density is everywhere finite may still only generate a small scattered field.

We have especially exploited ray theory, despite the fact that it only applies when the target and cloak are much larger in size than the incident wavelength and despite the existence of spatial nonuniformities. Fortunately, for the target geometries we have considered, exact eigenfunction expansions are available and we have shown how the Watson transformation can be used on these expansions so as to verify the applicability of ray theory.

Our analysis has not only predicted differences between the scattered fields for spherical targets and cloaks compared to cylindrical ones, but has also revealed that our regularisations create narrow shadow regions in the scattered field behind the target and enhanced insonification near the front of the target. Furthermore we have been able to study cloaks whose density is radially symmetric but quite different from the perfect cloaking density and insonification by a point source rather than a plane wave. 


\section{Acknowledgements}

We are very grateful to a referee for many useful comments and particularly for helping us to relate our work to the TO literature.

BDS would like to thank David Stocks for helpful discussions during the early stages of this work.

Also, all the authors would like to acknowledge the tremendously positive influence that Sam Howison has had over applied mathematics in recent decades and hope that this will continue for many years to come.

\section{References}

[1] S. A. Cummer, B-I. Popa, D. Schurig, D. R. Smith, J. Pendry, M. Rahm and A.Starr, Scattering Theory Derivation of a 3D Acoustic Shell. Phys. Rev. lett. 100,024301 (2008).

[2] A. Greenleaf, Y. Kurylev,M.Lassas and G. Uhlmann. Cloaking Devices, Electromagnetic Wormholes and Transformation Optics. SIAM Review, 51, 3-33, (2009).

[3] G. W. Milton, M.Briane and J. R. Willis, On cloaking for elasticity and physical equations with a transformation invariant form. New J. Physics. 8,article 248, (2006)

[4] A. N. Norris, Acoustic Cloaking Theory, Proc. R. Soc. A 464, 2411-2434,(2008).

[5] A. N. Norris, Acoustic cloaking in 2D and 3D using finite mass. arxiv.org/abs/0802.0701, (2008).

[6] A. N. Norris and A. Shuvalov, Elastic cloaking theory. Wave Motion 48, 525-538, (2011).

[7] G. Hu and H. Liu, Nearly cloaking the elastic wave fields. J. Math. Pure Appl. 104, 1045-1074, (2015).

[8] R. V. Kohn, H. Shen, M. S.Vogelius and M. I. Weinstein, Cloaking via change of variables in electric impedance tomography. Inverse Problems 24, 015-016, (2008).

[9] R. V. Kohn, D. Onofrei, M. S. Vogelius and M. I. Weinstein, Cloaking via change of variables in the Helmholtz equation. Comm. Pure Appl. Math. 63, 0973-1016, (2010).

[10] D. S. Jones, Acoustic and electromagnetic waves, Oxford University Press, (1986).

[11] E. A. Spence, The Watson transformation revisited. http:people.bath.ac.uk/eas25/Sp13b.pdf (2014).

[12] P. Gao and L. Wu, Non-singular acoustic cloak derived by the ray tracing method with rotationally symmetric transformations. Proc. Roy. Soc. Lond. (A) 472, 20150348 http://dx.doi.org/10.1098/rspa.2015.0348 (2016). 
[13] F. W. J. Olver et. al (eds) NIST Handbook of Mathematical Functions. Cambridge University Press, (2010).

[14] D.Colton and R. Kress. Inverse Acoustic and Electromagnetic Scattering Theory.Springer (2013).

[15] H. M. Nussenszveig. High-Frequency scattering by an impenetrable sphere. Annals of Physics 34 23-95 (1965).

[16] T.Tanaka, O.Matoba, Evolution and design of a large-scale cloaking device by the Hamilton-based ray-tracing method. J.Opt.Soc.Am.,34, 1052- 1059,(2017).

[17] M.M.Crosskey, A.T.Nixon, L.M.Schick, G.Kovacic, Invisibility Cloaking via nonsmooth Transformation Optics and Ray Tracing, Phys.Lett.A, 375, 1903-1911,(2011).

[18] C-Y.Hu, C-H.Lin, Reverse Ray Tracing for Transformation Optics. Opt.Exp., 23, $23017622(2015)$.

[19] J. Ockendon, S. Howison, A.Lacey and A. Movchan. Applied Partial Differential Equations, Oxford University Press (2003).

[20] J.R.Ockendon, R.H.Tew, Thin layer Solutions of the Helmholtz and Related Equations, SIAM Review, 54, 3-51,(2012).

[21] Y. Urzhumov, F. Ghezzo, J. Hunt and D. R. Smith. Acoustic cloaking transformations from attainable properties. New J. Phys, 12(7):073014, (2010).

[22] G. Dassios and R. E. Kleinman. Low-frequency Scattering, Oxford University Press (2000). 